\title{
DISEÑO DE UNA APLICACIÓN WEB PARA EL PROCESO EDUCATIVO SOBRE EL USO DEL LOGARITMO EN EL CAMPO DE LAS MATEMÁTICAS FINANCIERAS
DESIGN OF A WEB APPLICATION FOR THE EDUCATIONAL PROCESS ABOUT THE USE OF LOGARITHM IN THE FIELD OF FINANCIAL MATHEMATICS

\author{
Ricardo-Adán Salas-Rueda \\ Instituto de Ciencias Aplicadas y Tecnología, Universidad Nacional Autónoma de México, México \\ ricardo.salas@icat.unam.mx \\ Fernando Gamboa-Rodríguez \\ Instituto de Ciencias Aplicadas y Tecnología, Universidad Nacional Autónoma de México, México \\ fernando.gamboa@icat.unam.mx \\ Érika-Patricia Salas-Rueda \\ Instituto Tecnológico y de Estudios Superiores de Monterrey, México \\ serik_patt@hotmail.com \\ Rodrigo-David Salas-Rueda \\ Universidad Autónoma Metropolitana, México \\ rodrigodavidsalas@hotmail.com
}

RESUMEN: Esta investigación cuantitativa tiene como objetivo analizar el impacto de la Aplicación web para el proceso Educativo sobre el Logaritmo (AEL) por medio de la ciencia de datos y el aprendizaje automático (regresión lineal). La muestra está compuesta por 29 alumnos que cursaron la asignatura Matemáticas Básicas en una universidad mexicana durante el ciclo escolar 2015. La AEL presenta el procedimiento matemático sobre el uso del logaritmo para calcular el tiempo (número de años) durante la solicitud de un crédito bancario. El modelo TPACK permite organizar la AEL por medio del conocimiento tecnológico (lenguaje de programación PHP), conocimiento pedagógico (estrategia de simulación) y conocimiento disciplinar (uso del logaritmo en el campo de las matemáticas financieras). Los resultados del aprendizaje automático con 50\%, 60\% y $70 \%$ de entrenamiento indican que la AEL influye positivamente la asimilación del conocimiento sobre el logaritmo, el desarrollo de habilidades matemáticas y la comprensión sobre los conceptos teóricos del logaritmo en el campo de las matemáticas financieras. Asimismo, la ciencia de datos identifica tres modelos predictivos sobre el uso de la AEL en el proceso de enseñanza-aprendizaje. Por último, la tecnología está transformando la interacción entre los contenidos escolares y estudiantes durante el Siglo XXI.

PALABRAS CLAVE: tecnología; enseñanza superior; ciencia de datos; TIC; aprendizaje automático.

ABSTRACT: This quantitative research aims at analyzing the impact of the Web Application for the Educational Process on Logarithm (AEL) through data science and machine learning (linear regression). The sample is composed of 29 students who studied 
the Basic Mathematics course in a Mexican university during the 2015 school year. The AEL presents the mathematical procedure on the use of the logarithm to find the time (number of years) during the application for a bank loan. The TPACK model allows the organization of the AEL through technological knowledge (PHP programming language), pedagogical knowledge (simulation strategy) and disciplinary knowledge (use of the logarithm in the field of financial mathematics). The results of machine learning with $50 \%$, $60 \%$ and $70 \%$ training indicate that the AEL positively influences the assimilation of knowledge about the logarithm, development of mathematical skills and understanding of the theoretical concepts of the logarithm in the field of financial mathematics. Likewise, data science identifies three predictive models on the use of the AEL in the teachinglearning process. Finally, technology is transforming the interaction between the school content and students during the 21st century.

KEYWORDS: technology; higher education; data science; ICT; machine learning.

\section{Introducción}

Hoy en día, el proceso de enseñanza-aprendizaje está presentando cambios radicales debido a que los docentes están incorporando las Tecnologías de la Información y Comunicación (TIC) en las actividades escolares (BENZER; YILDIZ, 2019; HOUSTON, 2019; PYE; HOLT; SALZMAN, 2018). De hecho, los avances de la tecnología están provocando la aparición de nuevas estrategias educativas, modelos pedagógicos y escenarios virtuales de aprendizaje (BIDARRA; RUSMAN, 2017; MATOSAS-LÓPEZ; AGUADO-FRANCO; GÓMEZ-GALÁN, 2019; VOLK; COTIC; ZAJC; STARCIC, 2017).

Las herramientas digitales y los medios de comunicación están transformando la forma de acceso e interacción en los cursos (LÓPEZ-BELMONTE; POZO-SÁNCHEZ; PINO-ESPEJO, 2019). Por ejemplo, las aplicaciones web ofrecen flexibilidad de tiempo y espacio durante el proceso de enseñanza-aprendizaje (ORNELLAS; MUÑOZ-CARRIL, 2014).

Los dispositivos móviles están modificando la forma de acceder, consultar y usar los contenidos de los cursos (VOLK; COTIC; ZAJC; STARCIC, 2017; WARDANI; TOLLE; AKNURANDA, 2019). De hecho, los entornos virtuales educativos en Internet promueven el rol activo del estudiante (GALVIS; LÓPEZ; AARÓN, 2018). En particular, los juegos online incrementan la participación, motivación y satisfacción de los estudiantes durante el proceso de enseñanza-aprendizaje (FILIPPOU; CHEONG; CHEONG, 2018).

En el Siglo XXI, los docentes buscan mejorar la asimilación del conocimiento y desarrollar las habilidades de los estudiantes por medio de los juegos digitales, las aplicaciones web, el software educativo, los simuladores virtuales, los sistemas tutores inteligentes y las redes sociales (INFANTE-MORO; INFANTE-MORO; GALLARDOPÉREZ, 2019). Por consiguiente, las instituciones educativas está redefiniendo el proceso de enseñanza-aprendizaje por medio de las herramientas tecnológicas (SÁNCHEZLÓPEZ; PÉREZ-RODRÍGUEZ; FANDOS-IGADO, 2019).

Esta investigación cuantitativa propone el diseño y uso de la AEL en el proceso de enseñanza-aprendizaje sobre el logaritmo. Por lo tanto, las preguntas de investigación son:

- ¿Cuál es el impacto de la AEL en la asimilación del conocimiento sobre el 
logaritmo, el desarrollo de habilidades matemáticas y la comprensión sobre los conceptos teóricos del logaritmo en el campo de las matemáticas financieras?

- ¿Cuáles son los modelos predictivos sobre el uso de la AEL en el proceso de enseñanza-aprendizaje?

\section{Tecnología y modelo TPACK en el campo educativo}

Hoy en día, las instituciones educativas tienen el reto de actualizar las funciones de los docentes y mejorar las condiciones de enseñanza-aprendizaje (LÓPEZ-BELMONTE; POZO-SÁNCHEZ; FUENTES-CABRERA; GÓMEZ-GARCÍA, 2019; SÁNCHEZ-LÓPEZ; PÉREZ-RODRÍGUEZ; FANDOS-IGADO, 2019). En particular, las TIC adquieren un papel fundamental durante la planeación de los cursos (PYE; HOLT; SALZMAN, 2018; PRESTES-OLIVEIRA, 2019; STEVENS; GUO; LI, 2018). Asimismo, el uso de los modelos pedagógicos y tecnológicos como TPACK en el campo educativo está transformando la organización y realización de las actividades escolares en el Siglo XXI (PHILLIPS, 2016; SANCAR-TOKMAK; YANPAR-YELKEN, 2015).

\subsection{Uso de la tecnología en el proceso de enseñanza-aprendizaje}

Las herramientas tecnológicas como las aplicaciones online y los simuladores web mejoran el rendimiento académico de los estudiantes e incrementan la motivación dentro y fuera del salón de clases (ALAN; ZENGIN; KECECI, 2019). Incluso, la construcción de software educativo permite mejorar la calidad del proceso de enseñanza-aprendizaje (AN, 2019).

Diversos autores han utilizado las TIC en las actividades escolares con la finalidad de mejorar las condiciones de enseñanza-aprendizaje (ALAN; ZENGIN; KECECI, 2019; GALVIS; LÓPEZ; AARÓN, 2018; SALAS-RUEDA; LUGO-GARCÍA; RUIZ-SILVA, 2017). Por ejemplo, la incorporación de las TIC en la Maestría en Pedagogía facilitó la construcción de nuevos escenarios virtuales educativos y propició el rol activo de los estudiantes durante el proceso de enseñanza-aprendizaje (GALVIS; LÓPEZ; AARÓN, 2018). Incluso, Hrich, Lazaar y Khaldi (2019) explican que el sistema MaPSS adapta el escenario virtual educativo por medio de los agentes inteligentes. Del mismo modo, las redes sociales junto con las herramientas tecnológicas y las aplicaciones web permiten crear nuevos espacios que facilitan la asimilación del conocimiento y el desarrollo de las habilidades (SALAS-RUEDA; LUGO-GARCÍA; RUIZ-SILVA, 2017).

Las TIC permiten crear nuevos escenarios virtuales de aprendizaje y enseñanza por medio de la presentación, la recuperación, el intercambio, la producción y el almacenamiento de la información (ROMERO-GUTIÉRREZ; MARTÍNEZ-CHICO; JIMÉNEZ-LISO, 2018). Por ejemplo, el software Algodoo permite desarrollar las habilidades de los estudiantes en las áreas relacionadas con la ciencia, la tecnología, la ingeniería y las matemáticas por medio de la creación de simulaciones (ALAN; ZENGIN; KECECI, 2019). Asimismo, Salas-Rueda, Vázquez-Estupiñán y Lugo-García (2016) proponen la creación e implementación de una aplicación web con el objetivo de facilitar la asimilación del conocimiento sobre las derivadas y desarrollar las habilidades matemáticas de los estudiantes. 
Wardani, Tolle y Aknuranda (2019) construyeron el software Jariku con el propósito de mejorar las condiciones de enseñanza-aprendizaje en el nivel educativo básico por medio de los juegos digitales. Incluso, Hrich, Lazaar y Khaldi (2019) propone el uso de la inteligencia artificial durante la creación de aplicaciones educativas con la finalidad de personalizar el proceso de aprendizaje. En el campo de la estadística, Salas-Rueda (2019) construyó una aplicación web para facilitar la asimilación del conocimiento sobre la Distribución normal y desarrollar las habilidades matemáticas de los estudiantes.

Por último, el diseño, la construcción y la implementación de las herramientas digitales, las aplicaciones web y el software educativo están modificando la organización y planeación de las actividades escolares dentro y fuera del salón de clases (SALASRUEDA, 2018). Incluso, la incorporación de las TIC en el campo educativo permite el desarrollo de las competencias en los estudiantes (ALL; PLOVIE; NUÑEZ-CASTELLAR; LOOY, 2017; BRAY; TANGNEY, 2017; KERCKAERT; VANDERLINDE; BRAAK, 2015).

\subsection{Modelo TPACK}

El modelo TPACK (Technological Pedagogical And Content Knowledge) facilita la incorporación de las TIC en las actividades escolares por medio del uso de los conocimientos disciplinar, pedagógico y tecnológico (CABERO; BARROSO, 2016; SANCAR-TOKMAK; YANPAR-YELKEN, 2015). Este modelo proviene de las ideas propuestas por Shulman sobre la interacción de los conocimientos disciplinar y pedagógico (PHILLIPS, 2016; TURGUT; BOYLAN, 2017).

El conocimiento tecnológico (Technological Knowledge) se refiere al conocimiento sobre la tecnología (p.ej., uso de computadoras, pizarrones interactivos y proyectores) y el desarrollo de aplicaciones web y software educativo (TURGUT; BOYLAN, 2017). El conocimiento pedagógico (Pedagogical Knowledge) incluye el conocimiento sobre los procesos, métodos y prácticas de enseñanza-aprendizaje (CABERO; BARROSO, 2016; TURGUT; BOYLAN, 2017). Por último, el conocimiento disciplinar (Content Knowledge) se refiere al conocimiento sobre los contenidos del curso (DALAL; ARCHAMBAULT; SHELTON, 2017; TURGUT; BOYLAN, 2017). La interacción de estos conocimientos originan 7 componentes en el modelo TPACK: Tecnológico, Pedagógico, Disciplinar, Tecnológico-Pedagógico, Tecnológico-Disciplinar, Pedagógico-Disciplinar y TecnológicoPedagógico-Disciplinar (CENGIZ, 2015; DALAL; ARCHAMBAULT; SHELTON, 2017).

Hoy en día, los docentes utilizan el modelo TPACK para incorporar las herramientas tecnológicas en el campo educativo de manera eficiente (GÓMEZ, 2015). En el curso de Historia, el modelo TPACK fue utilizado para crear nuevas actividades y fomentar el rol activo de los estudiantes durante el proceso de enseñanza-aprendizaje (VAERENEWYCK; SHINAS; STECKEL, 2017). Por otro lado, los docentes de los cursos sobre el idioma inglés utilizaron el modelo TPACK durante la organización y creación de diversos contenidos audiovisuales (SANCAR-TOKMAK; YANPAR-YELKEN, 2015).

La interacción de los conocimientos tecnológicos, disciplinares y pedagógicos permiten la construcción de nuevos espacios educativos y la organización de creativas actividades dentro y fuera del salón de clases (BRANTLEY-DIAS; ERTMER, 2014; CENGIZ, 2015). Por lo tanto, los docentes y las instituciones educativas pueden apoyarse en el modelo TPACK para mejorar las condiciones de enseñanza-aprendizaje y actualizar las prácticas educativas por medio de la tecnología (DALAL; ARCHAMBAULT; SHELTON, 
2017).

\section{Método}

El objetivo de esta investigación cuantitativa es analizar el impacto de la AEL en el proceso de enseñanza-aprendizaje por medio de la ciencia de datos y el aprendizaje automático (regresión lineal). La AEL presenta el procedimiento matemático sobre el uso del logaritmo para calcular el tiempo (número de años) durante la solicitud de un crédito bancario.

\subsection{Participantes}

La muestra está compuesta por 29 alumnos, 15 hombres (51.72\%) y 14 mujeres (48.28\%), que cursaron la asignatura Matemáticas Básicas en una universidad mexicana durante el ciclo escolar 2015. Estos alumnos cursaron el primer semestre de las carreras en Administración $(n=7,24.14 \%)$, Contaduría ( $n=5,17.24 \%)$, Comercio $(n=11,37.93 \%)$ y Mercadotecnia $(n=6,20.69 \%)$.

\subsection{Procedimiento}

El procedimiento de esta investigación cuantitativa inició con la planeación, organización y construcción de la AEL, la cual está disponible en la siguiente dirección web: http://sistemasusables.com/aplicacion/logaritmo/inicio.html. El modelo TPACK permite organizar la AEL por medio del conocimiento tecnológico (lenguaje de programación PHP), conocimiento pedagógico (estrategia de simulación) y conocimiento disciplinar (uso del logaritmo en el campo de las matemáticas financieras). La Tabla 1 muestra el uso del modelo TPACK.

Tabla 1: Uso del modelo TPACK.

\begin{tabular}{|c|c|l|}
\hline No. & Conocimiento & \multicolumn{1}{|c|}{ Descripción } \\
\hline 1 & Tecnológico & El lenguaje de programación PHP permite la construcción de la AEL \\
\hline 2 & Pedagógico & $\begin{array}{l}\text { La estrategia de simulación permite mostrar a los estudiantes el } \\
\text { procedimiento detallado sobre el uso del logaritmo en el campo de las } \\
\text { matemáticas financieras }\end{array}$ \\
\hline 3 & Disciplinar & Uso del logaritmo en el campo de las matemáticas financieras \\
\hline 4 & Tecnológico-Pedagógico & $\begin{array}{l}\text { El lenguaje de programación PHP permite presentar el procedimiento } \\
\text { detallado sobre el uso del logaritmo en el campo de las matemáticas } \\
\text { financieras por medio de la estrategia de simulación }\end{array}$ \\
\hline 5 & Tecnológico-Disciplinar & $\begin{array}{l}\text { El lenguaje de programación PHP permite la construcción de la AEL } \\
\text { para transmitir el tema sobre el uso del logaritmo en el campo de las } \\
\text { matemáticas financieras }\end{array}$ \\
\hline 7 & Pedagógico-Disciplinar & $\begin{array}{l}\text { La estrategia de simulación es utilizada para mejorar el proceso de } \\
\text { enseñanza-aprendizaje sobre el uso del logaritmo en el campo de las } \\
\text { matemáticas financieras }\end{array}$ \\
\hline & Tecnológico- & Construcción de la AEL \\
\hline
\end{tabular}


El docente de la asignatura Matemáticas Básicas explicó los conceptos matemáticos sobre el uso del logaritmo (propiedades) en el salón de clases y utilizó la AEL como herramienta tecnológica de apoyo durante el proceso de enseñanzaaprendizaje. Los beneficios de esta aplicación web son la personalización del aprendizaje y la consulta de la información en cualquier momento y lugar.

La página de inicio en la AEL solicita la información sobre el monto, el capital y la tasa de interés para iniciar el procedimiento sobre el uso del logaritmo en el campo de las matemáticas financieras (Ver Figura 1).

\section{Aplicaciỏn dell logarituo}

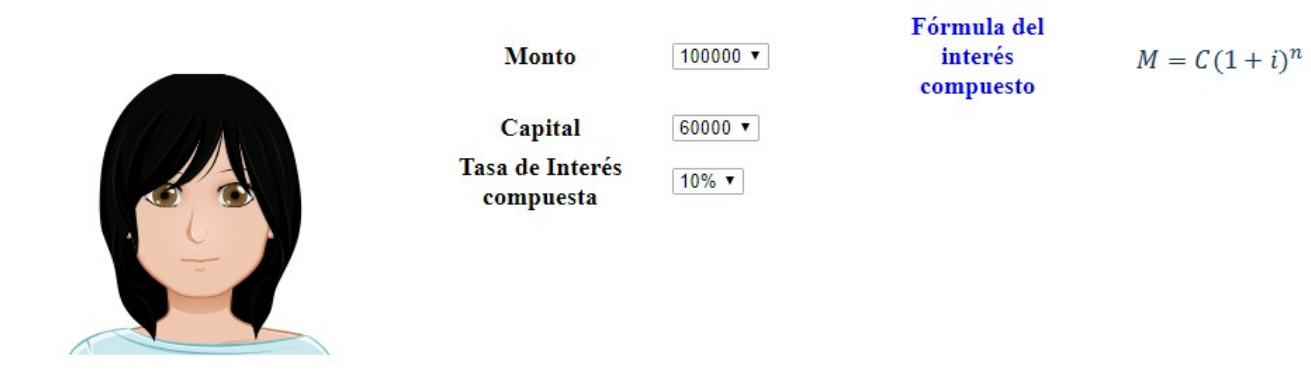

Proporciona los datos para encontrar

el número de años (n)

\section{Contimuar}

Figura 1: Página de inicio en la AEL.

Fuente: elaboración propia.

La AEL muestra el procedimiento detallado sobre el uso del logaritmo para calcular el tiempo (número de años) durante la solicitud de un crédito bancario (Ver Figura 2). 


\section{Aplicaciỏn dell logarituo}

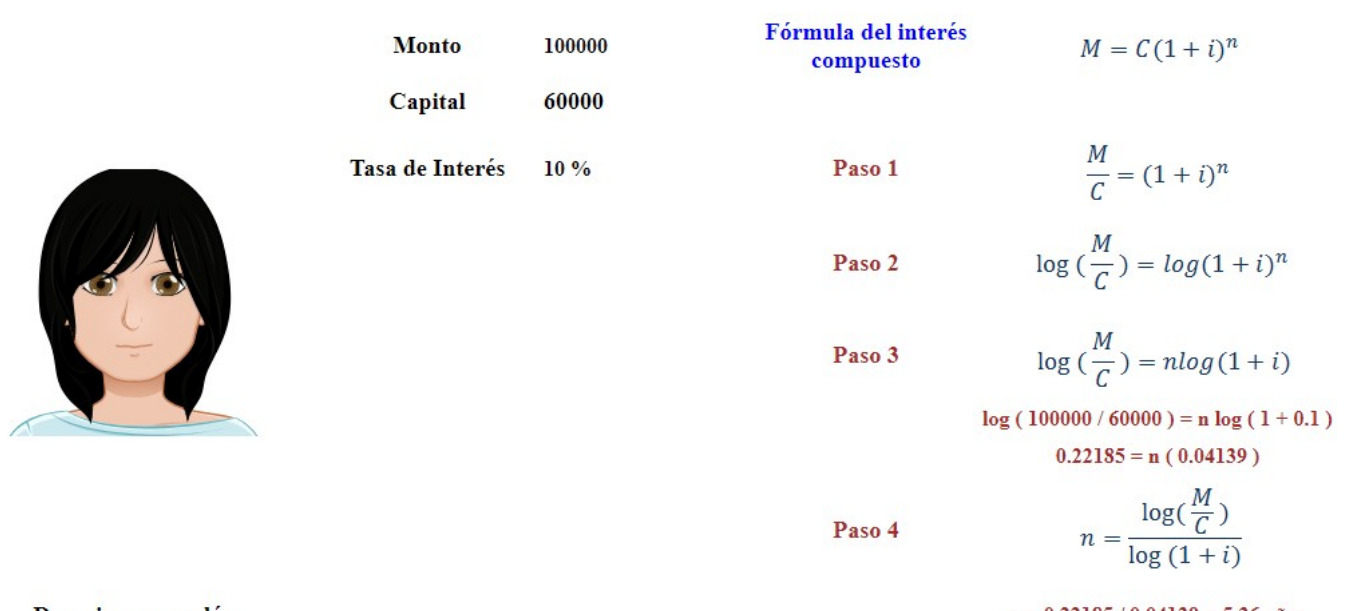

\section{Concimuar}

Figura 2: Cálculo sobre el número de años para el crédito bancario en la AEL.

Fuente: elaboración propia.

Las hipótesis de investigación relacionadas con el uso de la AEL en el proceso de enseñanza-aprendizaje son:

- Hipótesis $1(\mathrm{H} 1)$ : Los contenidos de la AEL influyen positivamente la asimilación del conocimiento sobre el logaritmo.

- Hipótesis $2(\mathrm{H} 2)$ : Los contenidos de la AEL influyen positivamente el desarrollo de habilidades matemáticas.

- Hipótesis $3(\mathrm{H} 3)$ : Los contenidos de la AEL influyen positivamente la comprensión sobre los conceptos teóricos del logaritmo en el campo de las matemáticas financieras.

La ciencia de datos permite identificar los siguientes modelos predictivos sobre el uso de la AEL en el campo educativo por medio de la técnica árbol de decisión:

- Modelo predictivo 1 sobre los contenidos de la AEL y la asimilación del conocimiento sobre el logaritmo.

- Modelo predictivo 2 sobre los contenidos de la AEL y el desarrollo de habilidades matemáticas.

- Modelo predictivo 3 sobre los contenidos de la AEL y la comprensión sobre los conceptos teóricos del logaritmo en el campo de las matemáticas financieras.

\subsection{Análisis de datos}

La herramienta Rapidminer es gratuita y permite realizar el cálculo del aprendizaje automático (regresión lineal) para evaluar las hipótesis sobre el uso de la AEL en el proceso de enseñanza-aprendizaje y construir los modelos predictivos por medio de la técnica árbol de decisión.

Con respecto al aprendizaje automático, la sección de entrenamiento (50\%, 60\% y 
$70 \%$ de la muestra) permite calcular las regresiones lineales y la sección de evaluación (50\%, 40\% y $30 \%$ de la muestra) permite identificar la exactitud de estas regresiones lineales.

Por otro lado, la ciencia de datos utiliza la información sobre el perfil del estudiante (edad, sexo y carrera) y el uso de la AEL en el proceso de enseñanza-aprendizaje para construir los modelos predictivos por medio de la técnica árbol de decisión.

\subsection{Recolección de datos}

El instrumento de medición está compuesto por 7 preguntas cerradas sobre el uso de la AEL en el proceso educativo (Ver Tabla 2). Este cuestionario fue aplicado al finalizar la unidad logaritmo durante el ciclo escolar 2015. Todos los alumnos de la asignatura Matemáticas Básicas contestaron el cuestionario.

Tabla 2: Cuestionario sobre el uso de la AEL.

\begin{tabular}{|c|c|c|c|c|c|c|}
\hline No. & Variable & Dimensión & Pregunta & Respuesta & $\mathrm{n}$ & $\%$ \\
\hline \multirow{15}{*}{1} & \multirow{15}{*}{ Estudiante } & \multirow{3}{*}{ Sexo } & \multirow[t]{3}{*}{ 1. Indica tu sexo } & & & \\
\hline & & & & Hombre & 15 & $51.72 \%$ \\
\hline & & & & Mujer & 14 & $48.28 \%$ \\
\hline & & \multirow{5}{*}{ Carrera } & \multirow[t]{5}{*}{ 2. Indica tu carrera } & & & \\
\hline & & & & Administración & 7 & $24.14 \%$ \\
\hline & & & & Contaduría & 5 & $17.24 \%$ \\
\hline & & & & Comercio & 11 & $37.93 \%$ \\
\hline & & & & Mercadotecnia & 6 & $20.69 \%$ \\
\hline & & \multirow{7}{*}{ Edad } & \multirow[t]{7}{*}{ 3. Indica tu edad } & & & \\
\hline & & & & 18 años & 17 & $58.62 \%$ \\
\hline & & & & 19 años & 6 & $20.69 \%$ \\
\hline & & & & 20 años & 1 & $3.45 \%$ \\
\hline & & & & 21 años & 2 & $6.90 \%$ \\
\hline & & & & 22 años & 1 & $3.45 \%$ \\
\hline & & & & 23 años & 2 & $6.90 \%$ \\
\hline \multirow{16}{*}{2} & \multirow{16}{*}{ Tecnología } & \multirow{4}{*}{ Contenidos } & \multirow{4}{*}{$\begin{array}{l}\text { 4. Los contenidos de la } \mathrm{AEL} \\
\text { facilitan el proceso de } \\
\text { aprendizaje }\end{array}$} & & & \\
\hline & & & & Bastante & 21 & $72.41 \%$ \\
\hline & & & & Mucho & 8 & $27.59 \%$ \\
\hline & & & & Poco & 0 & $0.00 \%$ \\
\hline & & \multirow{4}{*}{$\begin{array}{l}\text { Asimilación } \\
\text { del } \\
\text { conocimiento }\end{array}$} & \multirow{4}{*}{$\begin{array}{l}\text { 5. El uso de la AEL en las } \\
\text { actividades escolares facilita la } \\
\text { asimilación del conocimiento } \\
\text { sobre el logaritmo }\end{array}$} & & & \\
\hline & & & & Bastante & 13 & $44.83 \%$ \\
\hline & & & & Mucho & 15 & $51.72 \%$ \\
\hline & & & & Poco & 1 & $3.45 \%$ \\
\hline & & \multirow{4}{*}{$\begin{array}{l}\text { Desarrollo de } \\
\text { habilidades } \\
\text { matemáticas }\end{array}$} & \multirow{4}{*}{$\begin{array}{l}\text { 6. El uso de la AEL en las } \\
\text { actividades escolares facilita el } \\
\text { desarrollo de habilidades } \\
\text { matemáticas }\end{array}$} & & & \\
\hline & & & & Bastante & 17 & $58.62 \%$ \\
\hline & & & & Mucho & 12 & $41.38 \%$ \\
\hline & & & & Poco & 0 & $0.00 \%$ \\
\hline & & \multirow{4}{*}{$\begin{array}{l}\text { Contexto } \\
\text { práctico }\end{array}$} & \multirow{4}{*}{$\begin{array}{l}\text { 7. El uso de la AEL en las } \\
\text { actividades escolares facilita la } \\
\text { comprensión sobre los } \\
\text { conceptos teóricos del } \\
\text { logaritmo en el campo de las } \\
\text { matemáticas financieras }\end{array}$} & & & \\
\hline & & & & Bastante & 16 & $55.17 \%$ \\
\hline & & & & Mucho & 10 & $34.48 \%$ \\
\hline & & & & Poco & 3 & $10.34 \%$ \\
\hline
\end{tabular}

Fuente: elaboración propia. 


\section{Resultados}

A continuación, se presentan los resultados sobre el impacto de la AEL en el proceso de enseñanza-aprendizaje por medio de la ciencia de datos y el aprendizaje automático (regresión lineal).

Los contenidos de la AEL facilitan bastante $(n=21,72.41 \%)$ y mucho $(n=8,27.59 \%)$ el proceso de aprendizaje (Ver Tabla 2). Asimismo, los resultados del aprendizaje automático con 50\%, 60\% y $70 \%$ de entrenamiento indican que los contenidos de la AEL influyen positivamente la asimilación del conocimiento sobre el logaritmo, el desarrollo de habilidades matemáticas y la comprensión sobre los conceptos teóricos del logaritmo en el campo de las matemáticas financieras (Ver Tabla 3).

Tabla 3: Resultados del aprendizaje automático (regresión lineal).

\begin{tabular}{|c|c|c|c|c|}
\hline Hipótesis & Entrenamiento & Regresión lineal & Conclusión & Error cuadrado \\
\hline H1: Contenidos de la AEL & $50 \%$ & $\mathrm{y}=0.166 \mathrm{x}+1.333$ & Acepta: 0.166 & 0.200 \\
\cline { 2 - 5 } $\begin{array}{c}\rightarrow \text { asimilación del } \\
\text { conocimiento }\end{array}$ & $60 \%$ & $\mathrm{y}=0.288 \mathrm{x}+1.173$ & Acepta: 0.288 & 0.188 \\
\cline { 2 - 5 } & $70 \%$ & $\mathrm{y}=0.312 \mathrm{x}+1.125$ & Acepta: 0.312 & 0.176 \\
\hline \multirow{2}{*}{$\begin{array}{c}\text { H2: Contenidos de la AEL } \\
\rightarrow \text { desarrollo de } \\
\text { habilidades matemáticas }\end{array}$} & $50 \%$ & $\mathrm{y}=0.249 \mathrm{x}+1.166$ & Acepta: 0.249 & 0.223 \\
\cline { 2 - 5 } & $60 \%$ & $\mathrm{y}=0.115 \mathrm{x}+1.269$ & Acepta: 0.115 & 0.220 \\
\hline $\begin{array}{c}\text { H3: Contenidos de la AEL } \\
\rightarrow \text { comprensión sobre los } \\
\text { conceptos teóricos }\end{array}$ & $70 \%$ & $\mathrm{y}=0.187 \mathrm{x}+1.125$ & Acepta: 0.187 & 0.249 \\
\cline { 2 - 5 } & $60 \%$ & $\mathrm{y}=0.083 \mathrm{x}+1.166$ & Acepta: 0.083 & 0.854 \\
\hline
\end{tabular}

Fuente: elaboración propia.

\subsection{Asimilación del conocimiento}

El uso de la AEL en las actividades escolares facilita bastante ( $n=13,44.83 \%$ ), mucho $(n=15,51.72 \%)$ y poco $(n=1,3.45 \%)$ la asimilación del conocimiento sobre el logaritmo (Ver Tabla 2). Los resultados del aprendizaje automático con 50\% (0.166), 60\% (0.288) y 70\% (0.312) de entrenamiento indican que la hipótesis 1 es aceptada (Ver Tabla 3). Por lo tanto, los contenidos de la AEL influyen positivamente la asimilación del conocimiento sobre el logaritmo.

La Figura 3 muestra el modelo predictivo 1 sobre el uso de la AEL en el contexto educativo con la exactitud de $79.31 \%$. Por ejemplo, si el estudiante cursa la carrera de Administración y considera que los contenidos de la AEL facilitan bastante el proceso de aprendizaje entonces el uso de la AEL en las actividades escolares facilita bastante la asimilación del conocimiento sobre el logaritmo. 


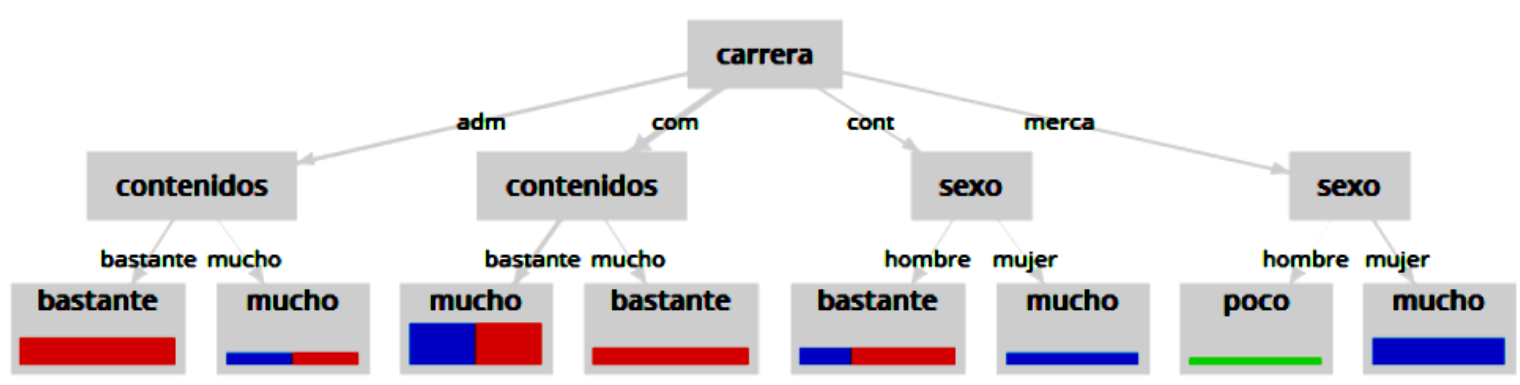

Figura 3: Modelo predictivo 1 sobre el uso de la AEL.

Fuente: elaboración por medio de la herramienta Rapidminer.

El modelo predictivo 1 tiene 4 condiciones sobre los contenidos de la AEL y la asimilación del conocimiento sobre el logaritmo. Por ejemplo, si el estudiante cursa la carrera de Comercio y considera que los contenidos de la AEL facilitan bastante el proceso de aprendizaje entonces el uso de la AEL en las actividades escolares facilita mucho la asimilación del conocimiento sobre el logaritmo (Ver Tabla 4).

Tabla 4: Modelo predictivo 1 sobre el uso de la AEL.

\begin{tabular}{|c|c|c|c|c|c|}
\hline No. & $\begin{array}{c}\text { Contenidos } \\
\text { de la AEL } \rightarrow \\
\text { aprendizaje }\end{array}$ & Carrera & Sexo & Edad & $\begin{array}{c}\text { Uso de la AEL } \rightarrow \text { asimilación del } \\
\text { conocimiento sobre el logaritmo }\end{array}$ \\
\hline 1 & Bastante & Administración & - & - & Bastante \\
\hline 2 & Mucho & Administración & - & - & Mucho \\
\hline 3 & Bastante & Comercio & - & - & Mucho \\
\hline 4 & Mucho & Comercio & - & - & Bastante \\
\hline 5 & - & Contaduría & Hombre & - & Mucho \\
\hline 6 & - & Contaduría & Mujer & - & Poco \\
\hline 7 & - & Mercadotecnia & Hombre & - & Mucho \\
\hline 8 & - & Mercadotecnia & Mujer & - & . \\
\hline
\end{tabular}

Fuente: elaboración propia por medio de la herramienta Rapidminer.

\subsection{Desarrollo de habilidades matemáticas}

El uso de la AEL en las actividades escolares facilita bastante $(n=17,58.62 \%)$ y mucho $(n=12,41.38 \%)$ el desarrollo de habilidades matemáticas (Ver Tabla 2). Los resultados del aprendizaje automático con 50\% (0.249), 60\% (0.115) y 70\% (0.187) de entrenamiento indican que la hipótesis 2 es aceptada (Ver Tabla 3). Por lo tanto, los contenidos de la AEL influyen positivamente el desarrollo de habilidades matemáticas.

La Figura 4 muestra el modelo predictivo 2 sobre el uso de la AEL en el contexto educativo con la exactitud de $79.31 \%$. Por ejemplo, si el estudiante tiene una edad $\leq 19.5$ años, es Hombre y considera que los contenidos de la AEL facilitan bastante el proceso de aprendizaje entonces el uso de la AEL en las actividades escolares facilita bastante el desarrollo de habilidades matemáticas. 


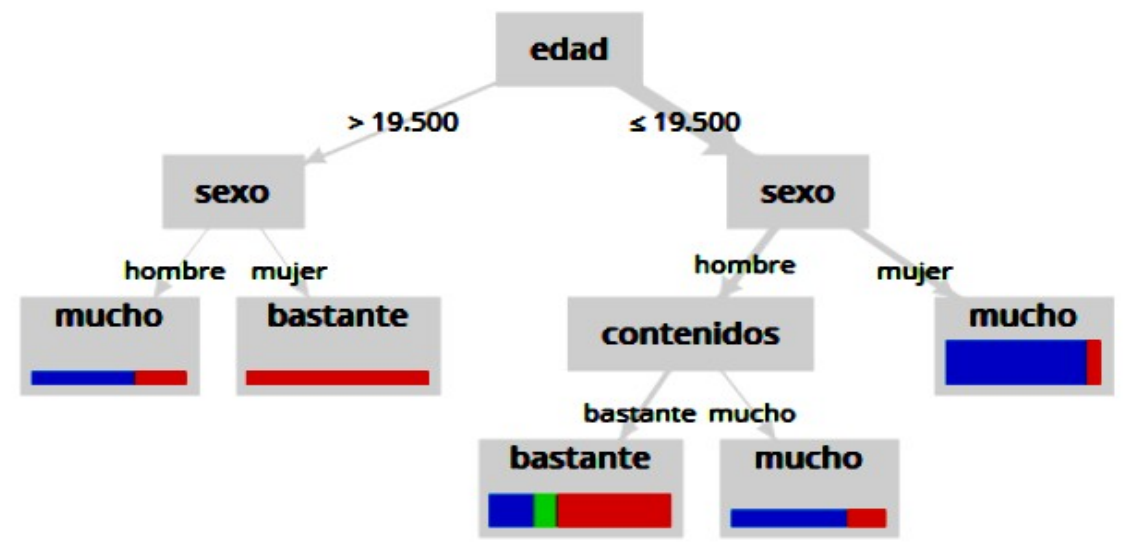

Figura 4: Modelo predictivo 2 sobre el uso de la AEL.

Fuente: elaboración por medio de la herramienta Rapidminer.

El modelo predictivo 2 tiene 2 condiciones sobre los contenidos de la AEL y el desarrollo de habilidades matemáticas. Por ejemplo, si el estudiante tiene una edad $\leq 19.5$ años, es Hombre y considera que los contenidos de la AEL facilitan mucho el proceso de aprendizaje entonces el uso de la AEL en las actividades escolares facilita mucho el desarrollo de habilidades matemáticas (Ver Tabla 5).

Tabla 5: Modelo predictivo 2 sobre el uso de la AEL.

\begin{tabular}{|c|c|c|c|c|c|}
\hline No. & $\begin{array}{c}\text { Contenidos de } \\
\text { la } A E L \rightarrow \\
\text { aprendizaje }\end{array}$ & Carrera & Sexo & Edad & $\begin{array}{c}\text { Uso de la AEL } \rightarrow \text { desarrollo de las } \\
\text { habilidades matemáticas }\end{array}$ \\
\hline 1 & Mucho & - & Hombre & $\leq 19.5$ años & Mucho \\
\hline 2 & Bastante & - & Hombre & $\leq 19.5$ años & Bastante \\
\hline 3 & - & - & Hombre & $>19.5$ años & Mucho \\
\hline 4 & - & - & Mujer & $>19.5$ años & Bastante \\
\hline 5 & - & - & Mujer & $\leq 19.5$ años & Mucho \\
\hline
\end{tabular}

Fuente: elaboración propia por medio de la herramienta Rapidminer.

\subsection{Comprensión sobre los conceptos teóricos en el contexto práctico}

El uso de la AEL en las actividades escolares facilita bastante $(n=16,55.17 \%)$, mucho $(n=10,34.48 \%)$ y poco $(n=3,10.34 \%)$ la comprensión sobre los conceptos teóricos del logaritmo en el campo de las matemáticas financieras (Ver Tabla 2). Los resultados del aprendizaje automático con 50\% (0.083), 60\% (0.192) y 70\% (0.062) de entrenamiento indican que la hipótesis 3 es aceptada (Ver Tabla 3). Por lo tanto, los contenidos de la AEL influyen positivamente la comprensión sobre los conceptos teóricos del logaritmo en el campo de las matemáticas financieras.

La Figura 5 muestra el modelo predictivo 3 sobre el uso de la AEL en el contexto educativo con la exactitud de $82.76 \%$. Por ejemplo, si el estudiante cursa la carrera de Comercio y considera que los contenidos de la AEL facilitan bastante el proceso de aprendizaje entonces el uso de la AEL en las actividades escolares facilita mucho la comprensión sobre los conceptos teóricos del logaritmo en el campo de las matemáticas financieras. 


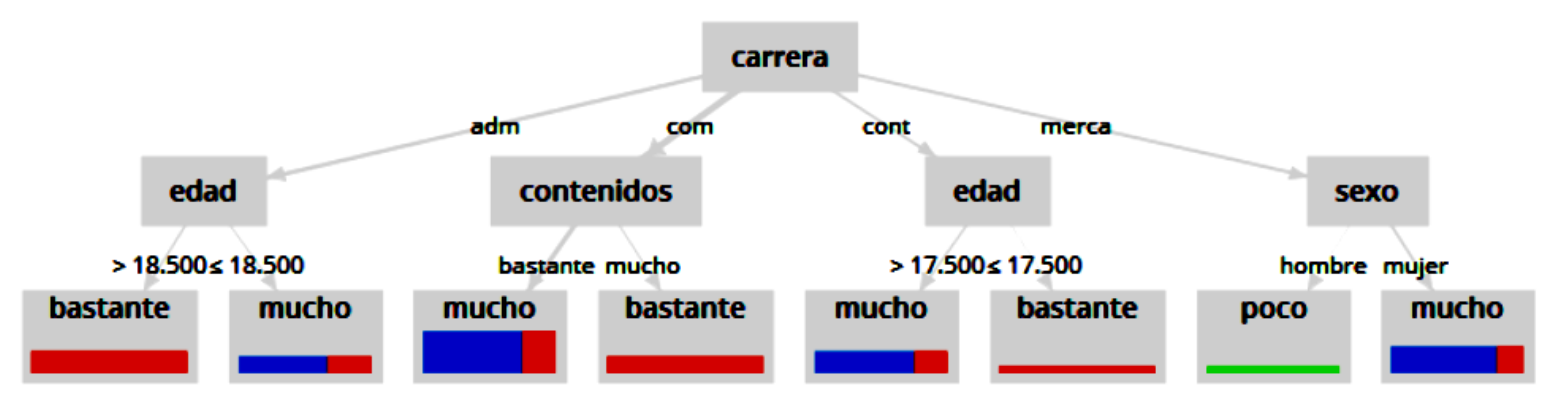

Figura 5: Modelo predictivo 3 sobre el uso de la AEL.

Fuente: elaboración por medio de la herramienta Rapidminer.

El modelo predictivo 3 tiene 2 condiciones sobre los contenidos de la AEL y la comprensión sobre los conceptos teóricos del logaritmo en el campo de las matemáticas financieras. Por ejemplo, si el estudiante cursa la carrera de Comercio y considera que los contenidos de la AEL facilitan mucho el proceso de aprendizaje entonces el uso de la AEL en las actividades escolares facilita bastante la comprensión sobre los conceptos teóricos del logaritmo en el campo de las matemáticas financieras (Ver Tabla 6).

Tabla 6: Modelo predictivo 3 sobre el uso de la AEL.

\begin{tabular}{|c|c|c|c|c|c|}
\hline No. & $\begin{array}{c}\text { Contenidos } \\
\text { de la AEL } \rightarrow \\
\text { aprendizaje }\end{array}$ & Carrera & Sexo & Edad & $\begin{array}{c}\text { Uso de la AEL } \rightarrow \text { comprensión } \\
\text { sobre los conceptos teóricos del } \\
\text { logaritmo en el contexto práctico }\end{array}$ \\
\hline 1 & Bastante & Comercio & - & - & Mucho \\
\hline 2 & Mucho & Comercio & - & - & Bastante \\
\hline 3 & - & Contaduría & - & $>17.5$ años & Mucho \\
\hline 4 & - & Contaduría & - & $\leq 17.5$ años & Bastante \\
\hline 5 & - & Administración & - & $>18.5$ años & Bastante \\
\hline 6 & - & Administración & - & $\leq 18.5$ años & Mucho \\
\hline 7 & - & Mercadotecnia & Hombre & - & Poco \\
\hline 8 & - & Mercadotecnia & Mujer & - & Mucho \\
\hline
\end{tabular}

Fuente: elaboración propia por medio de la herramienta Rapidminer.

\section{Discusión}

La tecnología está provocando grandes cambios durante la planeación y organización de las actividades escolares en el Siglo XXI (BRAY; TANGNEY, 2017; FILIPPOU; CHEONG; CHEONG, 2018; KERCKAERT; VANDERLINDE; BRAAK, 2015). En particular, está investigación cuantitativa analiza el impacto de la AEL en el proceso de enseñanza-aprendizaje por medio de la ciencia de datos y el aprendizaje automático.

El modelo TPACK permite crear nuevos espacios para el proceso de enseñanzaaprendizaje por medio del uso de las TIC. De hecho, los conocimientos tecnológico (lenguaje de programación PHP), pedagógico (estrategia de simulación) y disciplinar (uso del logaritmo en el campo de las matemáticas financieras) facilitaron la organización e implementación de la AEL. 


\subsection{Asimilación del conocimiento}

La mayoría de los estudiantes ( $n=15,51.72 \%)$ piensan que el uso de la AEL en las actividades escolares facilita mucho la asimilación del conocimiento sobre el logaritmo. Los resultados del aprendizaje automático sobre la hipótesis 1 son superiores a 0.160, por lo tanto, los contenidos de la AEL influyen positivamente la asimilación del conocimiento sobre el logaritmo. Asimismo, la ciencia de datos identifica 4 condiciones en el modelo predictivo 1 con la exactitud de $79.31 \%$.

\subsection{Desarrollo de habilidades matemáticas}

La incorporación de las herramientas tecnológicas permite el desarrollo de las habilidades en los estudiantes (ALL; PLOVIE; NUÑEZ-CASTELLAR; LOOY, 2017; KERCKAERT; VANDERLINDE; BRAAK, 2015). La mayoría de los estudiantes ( $\mathrm{n}=17$, $58.62 \%$ ) piensan que el uso de la AEL en las actividades escolares facilita bastante el desarrollo de habilidades matemáticas. Los resultados del aprendizaje automático sobre la hipótesis 2 son superiores a 0.110 , por lo tanto, los contenidos de la AEL influyen positivamente el desarrollo de habilidades matemáticas. Asimismo, la ciencia de datos identifica 2 condiciones en el modelo predictivo 2 con la exactitud de $79.31 \%$.

\subsection{Comprensión sobre los conceptos teóricos del logaritmo}

La tecnología permite innovar las actividades y prácticas escolares dentro y fuera del salón de clases (HOLBECK; HARTMAN, 2019; LÓPEZ-BELMONTE; POZOSÁNCHEZ; PINO-ESPEJO, 2019; ORNELLAS; MUÑOZ-CARRIL, 2014). La mayoría de los estudiantes ( $n=16,55.17 \%)$ piensan que el uso de la $A E L$ en las actividades escolares facilita bastante la comprensión sobre los conceptos teóricos del logaritmo en el campo de las matemáticas financieras. Los resultados del aprendizaje automático sobre la hipótesis 3 son superiores a 0.060 , por lo tanto, los contenidos de la AEL influyen positivamente la comprensión sobre los conceptos teóricos del logaritmo en el campo de las matemáticas financieras. Asimismo, la ciencia de datos identifica 2 condiciones en el modelo predictivo 3 con la exactitud de $82.76 \%$.

Las herramientas tecnológicas están transformando el acceso y uso de la información en el campo educativo (BIDARRA; RUSMAN, 2017; STEVENS; GUO; LI, 2018). En particular, los contenidos de la AEL influyen positivamente la asimilación del conocimiento sobre el logaritmo, el desarrollo de habilidades matemáticas y la comprensión sobre los conceptos teóricos del logaritmo en el campo de las matemáticas financieras.

\section{Conclusión}

Las funciones de los docentes están cambiando debido a la incorporación de las aplicaciones web, las herramientas digitales y el software educativo en el proceso de enseñanza-aprendizaje. En particular, la AEL presenta el procedimiento matemático sobre 
el uso del logaritmo para calcular el tiempo (número de años) durante la solicitud de un crédito bancario.

Los resultados del aprendizaje automático (regresión lineal) indican que la AEL influye positivamente la asimilación del conocimiento sobre el logaritmo, el desarrollo de habilidades matemáticas y la comprensión sobre los conceptos teóricos del logaritmo en el campo de las matemáticas financieras. Asimismo, la técnica árbol de decisión identifica tres modelos predictivos sobre el uso de la AEL en el campo educativo con una exactitud mayor a $79.30 \%$.

Por otro lado, el modelo TPACK facilita la planeación y organización de nuevas experiencias educativas por medio de los conocimientos tecnológico, disciplinar y pedagógico. De hecho, el lenguaje de programación PHP (conocimiento tecnológico), la estrategia de simulación (conocimiento pedagógico) y el uso del logaritmo en el campo de las matemáticas financieras (conocimiento disciplinar) permiten innovar el proceso educativo por medio de la construcción de la AEL.

Las limitaciones de esta investigación cuantitativa están relacionadas con el tamaño de la muestra y los contenidos de la AEL sobre el uso del logaritmo. Por lo tanto, las futuras investigaciones pueden construir aplicaciones web relacionadas con el uso de las funciones exponenciales, lineales, cuadráticas y racionales. Asimismo, los docentes tienen la oportunidad de crear los contenidos audiovisuales considerando diversos idiomas.

Esta investigación cuantitativa recomienda el diseño y la implementación de las aplicaciones web en el campo educativo con la finalidad de lograr flexibilidad de tiempo y espacio durante el proceso de enseñanza-aprendizaje. Las implicaciones de esta investigación permiten motivar la construcción de nuevos escenarios educativos por medio de la tecnología.

En conclusión, las instituciones educativas pueden actualizar las actividades escolares dentro y fuera del salón de clase por medio de las herramientas tecnológicas. Por ejemplo, los contenidos de la AEL facilitan la asimilación del conocimiento sobre el logaritmo, el desarrollo de habilidades matemáticas y la comprensión sobre los conceptos teóricos del logaritmo en el campo de las matemáticas financieras.

\section{Referencias}

ALAN, B.; ZENGIN, F. K.; KECECI, G. Using stem applications for supporting integrated teaching knowledge of pre-service science teachers. Journal of Baltic Science Education, v. 18, n. 2, p. 158-170, 2019.

ALL, A.; PLOVIE, B.; NUÑEZ-CASTELLAR, E. P.; LOOY, J. V. Pre-test influences on the effectiveness of digital-game based learning: A case study of a fire safety game. Computers \& Education, v. 114, p. 24-37, 2017.

AN, L. Application of Computer Technology in Aesthetic Education and Feature Analysis. International Journal of Emerging Technologies in Learning, v. 14, n. 14, p. 46-55, 2019.

BENZER, A. I.; YILDIZ, B. The effect of computer-aided 3d modeling activities on pre- 
service teachers' spatial abilities and attitudes towards $3 \mathrm{~d}$ modeling. Journal of Baltic Science Education, v. 18, n. 3, p. 335-348, 2019.

BIDARRA, J.; RUSMAN, E. Towards a pedagogical model for science education: bridging educational contexts through a blended learning approach. Open Learning: The Journal of Open, Distance and e-Learning, v. 32, n. 1, p. 6-20, 2017.

BRANTLEY-DIAS, L.; ERTMER, P. A. Goldilocks and TPACK: Is the Construct 'Just Right?' Journal of Research on Technology in Education, v. 46, n. 2, p. 103-128, 2014.

BRAY, A.; TANGNEY, B. Technology usage in mathematics education research - A systematic review of recent trends. Computers \& Education, v. 114, p. 255-273, 2017.

CABERO, J.; BARROSO, J. ICT teacher training: a view of the TPACK model. Culture and Education, v. 28, n. 3, p. 633-663, 2016.

CENGIZ, C. The development of TPACK, Technology Integrated Self-Efficacy and Instructional Technology Outcome Expectations of pre-service physical education teachers. Asia-Pacific Journal of Teacher Education, v. 43, n. 5, p. 411-422, 2015.

DALAL, M; ARCHAMBAULT, L; SHELTON, C. Professional Development for International Teachers: Examining TPACK and Technology Integration Decision Making. Journal of Research on Technology in Education, v. 49, n. 3, p. 117-133, 2017.

FILIPPOU, J.; CHEONG, C.; CHEONG, F. A Model to Investigate Preference for Use of Gamification in a Learning Activity. Australasian Journal of Information Systems, v. 22, p. 1-23, 2018.

GALVIS, Á. H.; LÓPEZ, Y. Y; AARÓN, M. A. Learning from a transformation of pedagogical practices in master's degree courses in the hybrid mode of learning. Revista de Educación a Distancia, v. 58, n. 2, p. 1-35, 2018.

GÓMEZ, M. When Circles Collide: Unpacking TPACK Instruction in an Eighth-Grade Social Studies Classroom. Computers in the Schools, v. 32, n. 3, p. 278-299, 2015.

HOLBECK, R.; HARTMAN, J. Efficient Strategies for Maximizing Online Student Satisfaction: Applying Technologies to Increase Cognitive Presence, Social Presence, and Teaching Presence. Journal of Educators Online, v. 15, n. 3, p. 1-6, 2019.

HOUSTON, L. Efficient Strategies for Integrating Universal Design for Learning in the Online Classroom. Journal of Educators Online, v. 15, n. 3, p. 1-16, 2019.

HRICH, N.; LAZAAR, M.; KHALDI, M. MAPSS: An Intelligent Architecture for the Pedagogical Support. International Journal of Emerging Technologies in Learning, v. 14, $\mathrm{n}$. 14, p. 19-30, 2019.

INFANTE-MORO, A; INFANTE-MORO, J.; GALLARDO-PÉREZ, J. The Importance of ICTS 
for Students as a Competence for their Future Professional Performance: the Case of the Faculty of Business Studies and Tourism of the University of Huelva. Journal of New Approaches in Educational Research, v. 8, n. 2, p. 201-213, 2019.

KERCKAERT, S.; VANDERLINDE, R.; BRAAK, J. V. The role of ICT in early childhood education: Scale development and research on ICT use and influencing factors. European Early Childhood Education Research Journal, v. 23, n. 2, p. 183-199, 2015.

LÓPEZ-BELMONTE, J.; POZO-SÁNCHEZ, S.; FUENTES-CABRERA, A.; GÓMEZGARCÍA, G. Análisis del efecto de la formación b-learning en el profesorado. Estudio de caso de una cooperativa de enseñanza. Texto Livre: Linguagem e Tecnologia, v. 12, n. 2, p. $98-115,2019$.

LÓPEZ-BELMONTE, J.; POZO-SÁNCHEZ, S.; PINO-ESPEJO, M. J. Projection of the Flipped Learning Methodology in the Teaching Staff of Cross-Border Contexts. Journal of New Approaches in Educational Research, v. 8, n. 2, p. 184-200, 2019.

MATOSAS-LÓPEZ, L.; AGUADO-FRANCO, J. C.; GÓMEZ-GALÁN, J. Constructing an Instrument with Behavioral Scales to Assess Teaching Quality in Blended Learning Modalities. Journal of New Approaches in Educational Research, v. 8, n. 2, p. 142-165, 2019.

ORNELLAS, A.; MUÑOZ-CARRIL, P. C. A methodological approach to support collaborative media creation in an e-learning higher education context. Open Learning: The Journal of Open, Distance and e-Learning, v. 29, n. 1, p. 59-71, 2014.

PHILLIPS, M. Re-contextualising TPACK: exploring teachers' (non-) use of digital technologies. Technology, Pedagogy and Education, v. 25, n. 5, p. 555-571, 2016.

PRESTES-OLIVEIRA, J. ICT and children's literature: challenges of pedagogical practice in the digital age. Texto Livre: Linguagem e Tecnologia, v.12, n. 2, p. 116-134, 2019.

PYE, G.; HOLT, D.; SALZMAN, S. Investigating different patterns of student engagement with blended learning environments in Australian business education: Implications for design and practice. Australasian Journal of Information Systems, v. 22, p. 1-23, 2018.

ROMERO-GUTIÉRREZ, M.; MARTÍNEZ-CHICO, M.; JIMÉNEZ-LISO, M. R. Online forum analysis of a joint master's degree by remote classes in terms of emotions. Revista de Educación a Distancia, v. 58, p. 1-21, 2018.

SALAS-RUEDA, R. A. Uso del servicio en la nube GeoGebra durante el proceso enseñanza-aprendizaje sobre las matemáticas. Revista iberoamericana para la investigación y el desarrollo educativo, v. 8, p. 23-52, 2018.

SALAS-RUEDA, R. A. Construction and evaluation of a web application for the educational process on Normal Distribution considering the science of data and machine learning. Research in Learning Technology, v. 27, p. 1-24, 2019. 
SALAS-RUEDA, R. A.; LUGO-GARCÍA, J. L.; RUIZ-SILVA, H. F. Perspectivas de los estudiantes sobre el uso de redes sociales en el proceso educativo superior. Vivat academia, n. 139, p. 53-66, 2017.

SALAS-RUEDA, R. A.; VÁZQUEZ-ESTUPIÑÁN, J. J.; LUGO-GARCÍA, J. L. Uso del avatar en el proceso de enseñanza-aprendizaje sobre las aplicaciones de las derivadas. Revista de Comunicación de la SEECI, v. 39, p.71-84, 2016.

SANCAR-TOKMAK, H.; YANPAR-YELKEN, T. Effects of creating digital stories on foreign language education pre-service teachers' TPACK self-confidence. Educational Studies, v. 41, n. 4, p. 444-461, 2015.

SÁNCHEZ-LÓPEZ, I.; PÉREZ-RODRÍGUEZ, A.; FANDOS-IGADO, M. Com-educational Platforms: Creativity and Community for Learning. Journal of New Approaches in Educational Research, v. 8, n. 2, p. 214-226, 2019.

STEVENS, K. J.; GUO, Z.; LI, Y. Typology and Hierarchy of Students' Motivations to Use Technology in Learning. Australasian Journal of Information Systems, v. 22, p. 1-30, 2018.

TURGUT, Y.; BOYLAN, M. Tracing preservice English language teachers' perceived TPACK in sophomore, junior, and senior levels. Cogent Education, v. 4, n. 1, p. 1-20, 2017.

VAERENEWYCK, L. M.; SHINAS, V. H.; STECKEL, B. Sarah's Story: One Teacher's Enactment of TPACK + in a History Classroom. Literacy Research and Instruction, v. 56, n. 2, p. 158-175, 2017.

VOLK, M.; COTIC, M.; ZAJC, M.; STARCIC, A. I. Tablet-based cross-curricular maths vs. traditional maths classroom practice for higher-order learning outcomes. Computers \& Education, v. 114, p. 1-23, 2017.

WARDANI, I.; TOLLE, H.; AKNURANDA, I. Evaluation of an Educational Media on Cube Nets Based on Learning Effectiveness and Gamification Parameters. International Journal of Emerging Technologies in Learning, v. 14, n. 14, p. 4-17, 2019.

Recebido em dia 19 de outubro de 2019. Aprovado em dia 13 de março de 2020. 